\title{
Chinese food additives enterprises transformation strategy
}

\author{
Yuhan Zhang ${ }^{1, a}$, Yiming Shao ${ }^{2, b}$ \\ ${ }^{1}$ School of Nanjing University Of Science And Technology, Nanjing 210000, China; \\ ${ }^{2}$ School of Nanjing University Of Science And Technology, Nanjing 210000, China. \\ a644203486@qq.com, bsym171@163.com
}

Keywords: Food additives, Industrial concentration, Brand, Enterprise transformation.

\begin{abstract}
At present, with the economic progress brought about by the improvement of living standards, scientific and technological progress and the concept of the Internet + , the domestic demand has led to the rapid growth of the food industry. Among them, food additives, as a key part of the food industry, have also made great strides in development. However, due to the food safety incidents caused by imperfect regulation, the industrial structure is difficult to centralized management, industry mixed business unfair competition and consumers panic psychology makes the food additive industry development and business transformation face some difficulties. This article analyzes the current situation of the development of domestic food additive industry, and on the basis of the current situation and trends of the development, the transition strategy for the food additives enterprises in transition period is given.
\end{abstract}

\section{Introduction}

Chinese food additive industry has enjoyed a good momentum of growth. The efficiency of Chinese food additives industry has been steadily increasing. The average annual growth rate of food additives is about 15\%, higher than the world average. The total output value of Chinese food additive industry surpassed 100 billion yuan in 2015, this industry has broad prospects [1]. In decades of growth, the food industry has made progress in technology and new product development, the food additive industry has been integrated to a certain degree of concentration. There have been some mergers and acquisitions of large enterprises with economic power to acquire small and medium-sized enterprises, and the use of brand strategy in the industry to form a competitive strength. However, the overall concentration is still low, which greatly limits the future growth of food additives business space. In addition, due to limited technology development, there are about 1,500 kinds of food additives that can be used in China, far lower than the 2922 kinds that can be used in the United States [2]. This makes our country's food additives product categories relatively single, the developed countries in the world continue to research and development of technology has been gradually sub-varieties of specialized production, to further expand the international market share. Our country has no competitive advantage in producing only varieties and major categories. Food additives with higher demand for technology still need to import. To break this situation, we have higher requirements for independent innovation and research capabilities. With the further amendment of the food safety regulations, the supervision and inspection of quality is becoming more and more powerful. Corporate transformation is imminent. 


\section{Food additives industry trends}

Industrial concentration is increasingly strengthened. Industrial concentration is the current trend of the international industrial market, of course, including food additives industry. Low industry thresholds and the participation of foreign international companies make a large number of food additives enterprises in our country and scattered widely, intense competition caused by low concentration, making it difficult for enterprises to form economies of scale. This is a key factor in the lack of a globally competitive and well-known Chinese food additive business. Scattered brand market is difficult to truly meet the needs of consumers, and only those with economic strength, leading technology companies to achieve consumer acceptance and loyalty.

Towards the development of natural food additives. Today, food safety problems continue to occur, the use of illegal food additives caused by the vicious incident that make the public to chemical synthetic food additives demonized. Although it is in conformity with laws and regulations and the use of additives according to the dose standard is safe, non-toxic and harmless. However, this cognition has not been extensively publicized in science so that consumers are greatly misunderstood in this area. So in the future for a long period of time, natural food additives will always be an irresistible trend of development, and will radiate the entire industry.

The supervision of the safe use of food additives is more and more strict. At present, in the process of food processing, the use of illegal additives in medium and large-sized enterprises has been controlled, but the use of excessive doses still exists. In particular, small businesses and workshops, which are difficult to regulate due to their small size and high mobility, have become the " convention " for overusing food additives in the industry. Poor supervision makes these workshops a chance, which is an important reason for the failure to cure food safety problems. In recent years, the government has continuously perfected the legal system of food additive industry and strictly regulated the addition of illegal food additives and abuse of food additives [3].

The development of modern biotechnology. The market share of our country and developing countries is less than one quarter in the industry. The lack of technology is the key reason for the limited development in our country. In particular, the research and development in the direction of food additive subdivision has been continuously occupied by the international market. Our country can only rely on imports in the case of backward technology. Chinese food additive industry has been making efforts to break this stalemate. In recent years, capital investment and equipment renewal have been greatly increased, which has guaranteed the development of modern biotechnology.

Brand of food additives. One of the most obvious reactions after industrial concentration is the branding of enterprises to increase market share. Brand food additives companies in order to maintain their good corporate image, will be bound by their own bad behavior, and urge enterprises to focus on the long-term development of enterprises, rather than short-term private interests to undermine the interests of consumers. Due to the effect of brand endorsement, the product quality and safety of food additives companies will be more assured [4]. Additives corporate branding is also beneficial to government direct management and consumer supervision.

\section{Problems of Chinese food additives industry}

There are few high-tech types and the dependence on imported technology and equipment leads to a lack of competitiveness in the world. China's food additives industry occupies a low market share, and the developed countries in the world are not only technologically advanced, but also have been favored by manufacturers for targeted R \& D and production in the direction of food 
additive subdivision. Compared with the European and American markets, our country lacks competitiveness.

Industry regulations are imperfect, supervision and management are inadequate, and there is unfair competition in the industry. Food safety has always been the focus of attention. However, the vicious incidents caused by the use of illegal food additives have taken place. This not only undermines the interests of competitors and disrupts the order of food markets, but also makes consumers do not trust the additive industry.

The industrial concentration is low, lacking big brands that can enter the international market. The relatively low threshold of Chinese food additive industry leading the fierce competition makes it difficult for any enterprise to take a leading position in the industry. Competition between brands, there is not enough scale and popularity to enter the international market.

\section{Chinese food additives enterprises transformation strategy}

Technological and management innovation. At present, there is a gap in China's food additive technology, there is no new product research and cost advantages in foreign exports. And the current traditional process technology has been difficult to meet the diverse needs of consumers, especially for functional health products and natural food additives needs. In order to solve the problem of limited development of enterprises, it is necessary for enterprises to increase capital investment in biotechnology, introduce professional and technical personnel to set up research teams, and constantly pay attention to changes in the market environment so as to provide customers with personalized services. Innovate the management methods, and introduce Informatization into the food additive industry to digitize management. Informatization means that enterprises digitalized management from raw material purchase to production. ERP system is used to simplify manual operation, which is conducive to scientific decision [5].

Green marketing. Food additive factories in the production process to continue to solve the problem of waste pollution, waste of resources, which greatly increased the operating costs of enterprises. The concept of green marketing is to meet the needs of consumers under the premise of the pursuit of sustainable development of enterprises and the environment put forward. Green Marketing Strategy Green products and green management require the necessary resources, but businesses can get a higher marginal profit when they get the green logo. With the popularity of green food, there is a sense of urgency in the green marketing of the food additive industry.

"Internet +" strategic transformation. As a traditional enterprise, food additives enterprises try to combine the "Internet +" is a new idea of the current transformation of food additives enterprises. In the era of big data security, it is not only a way for the government to manage and supervise to safeguard the fair competition in the industry, but also make food additives enterprises to establish a credit system for enterprises to bring a large number of customer resources and increase sales. Since the upstream enterprises of food additives do not directly contact with consumers, they adopt the B2B model to release product information through the online platform, and enterprises can freely trade, buy, auction, and pay online on the platform directly [6]. On the platform, there will be an enterprise credit rating evaluation and a financial supervision system to increase corporate reputation and operational efficiency. Downstream food additives companies launched a food additives query app and used the way of network tweet, consumers can always check the additives ingredients and dose ratio to enhance the knowledge of food additives to reduce the food additives are all toxic and harmful misunderstanding. 


\section{Summary}

In recent years, Chinese food additive industry has gained momentum and great potential. However, there are still some shortcomings in terms of technology, scale and quality control. To promote industrial concentration is the only way, food additives companies need to do with the current development trend of enterprise restructuring strategy.

\section{References}

[1] Information on http: //www.chyxx.com/industry/201607/431689.html.

[2] Fangcun Lan, Ye Li, Jie Lin. Research status of food additives in developed countries [J] .Guangdong Journal Of Trace Elements Science, 2013,20 (2).

[3] Linglin Jiang, Yuyang Li. Review of the status quo of China's food additives management [J]. China Health Policy Research, 2011 (07): 35.

[4] Rong Miao. Brand food additives companies will benefit from remediation [N]. China Food Quality News, 2012 (A02).

[5] Hanyan Chen. Discussion and Research on the Strategy of Reformation for Food Manufacturing Enterprise Informatization [J]. Food Industry, 2013,34 (01).

[6] Information on http: //news.xhby.net/system/2015/04/09/024288372.shtml 